\title{
Differences in Executive Functions applied to memory processes in people with Migraine: a Cross-Sectional Study*
}

\section{Diferencias en Funciones Ejecutivas aplicados a procesos de memoria} en personas con migraña: estudio transversal

\author{
Maria Fernanda Quiroz-Padilla \\ Patricia Pitta \\ LaUra LOMBANA-Angel \\ Laboratorio de Bases Biológicas del Comportamiento \\ Facultad de Psicología. Universidad de la Sabana, Bogotá, Colombia

\section{Gordon P.D. InGRAM} \\ Departamento Psicología \\ Universidad de los Andes, Bogotá, Colombia \\ Carolina Gómez \\ Independent Clinical Neuropsychologist, Bucaramanga, Colombia \\ Jorge A Restrepo \\ Clínica Universidad de la Sabana, Colombia \\ Facultad de Medicina. Universidad de la Sabana, Bogotá, Colombia
}

DOI: $10.11144 / J a v e r i a n a . u p s y 15-5$. defa

Received: 25 August 2016 | Accepted: 28 September 2016

a Corresponding author:
mariaqp@unisabana.edu.co

Para citar este artículo: Quiroz-Padilla, M. F., Pitta, P., Lombana-Angel, L., Ingram, P. D, Gómez, C., \& Restrepo, J. A.. (2016). Differences in Executive Functions applied to memory processes in people with Migraine: a CrossSectional Study. Universitas Psychologica, 15(5). http:// dx.doi.org/10.11144/Javeriana.upsy15-5.defa

\begin{abstract}
The aim of this cross-sectional study was to identify differences in memory processes and the role of executive functions in memory, in people with migraine and in a control group. Neuropsychological evaluation was made in one session on 63 participants distributed into interictal migraine-withaura $(\mathrm{n}=24)$, interictal migraine-without-aura $(\mathrm{n}=16)$ and control $(\mathrm{n}=23)$ groups. ANOVAs on the individual tasks revealed statistically significant differences between groups on Rey-Osterrieth direct and percentile copy strategy and recall (both $\mathrm{p}<0.001$ ). Differences were identified between control and migraine groups in performance on the Rey-Osterrieth Complex Figure, but not on other memory tasks, suggesting the existence of brain dysfunctions in people with migraine affecting organizational and planning abilities that are necessary for visual memory.

Keywords

Aura; executive functions; memory; migraine.
\end{abstract}

\section{RESUMEN}

El objetivo de este estudio transversal fue identificar posibles diferencias en los procesos de memoria y el rol de las funciones ejecutivas que se requieren para dicho proceso, en personas con diagnóstico de migraña y un grupo control. La evaluación neuropsicológica se realizó en una sesión en 63 participantes distribuidos en grupos de migraña con aura ( $\mathrm{n}$ $=24)$ y $\sin$ aura $(\mathrm{n}=16)$ —ambos grupos en periodo interictal- y un grupo control $(\mathrm{n}=23)$. El análisis de varianza en las pruebas individuales realizadas identifica una diferencia estadísticamente significativa entre los grupos en las estrategias de recobro y copia de la ejecución directa y percentil de la prueba de Figura compleja de Rey Osterrieth $(\mathrm{p}<0.001)$. 
Las diferencias entre los grupos migraña y el control en la Figura compleja de Rey Osterrieth, pero no en las otras pruebas de memoria, sugieren la existencia de una posible disfunción cerebral en personas con migraña que afecta las habilidades de organización y planeación necesarias para la memoria visual.

Palabras clave

Aura; funciones ejecutivas; memoria; migraña.

\section{Íntroduction}

Migraine is a complex and debilitating neurobiological disorder which is known as a primary headache and is characterized by several symptoms during intermittent and recurrent attacks (Borsook, Maleki, \& Burstein, 2015; Harmon, 2015; Ravishankar \& Demakis, 2007). Its prevalence is approximately 10 to $12 \%$ of the adult population (Ravishankar \& Demakis, 2007), leading to its classification in the top 20 most disabling diseases worldwide according to the World Health Organization (Finocchi \& Strada, 2014). Migraine is more than twice as common in females as in males, but it is still poorly understood whether there are sex differences in migraine's effects on cognition (Faria et al., 2015; Finocchi \& Strada, 2014; Maleki et al., 2012).

The most common symptoms of migraine are painful headache (unilateral and pulsating pain in most cases) which can last 4 hours or more without treatment; nausea/ vomiting; sensory deficits such as photophobia or phonophobia; and in some cases neurological alterations (Borsook et al., 2015; Bussone, 2004). Considering all this, migraine can have a disabling influence on the individual suffering from it. This disorder may impair the physical, cognitive, social or occupational dimensions of a patient's life, affecting many of their functional outcomes. Migraine can be an acute or a chronic disease. Its main classification is into migraine with aura (MWA) and migraine without aura (MWoA), with aura being defined as a distinct sensation, typically visual or auditory, that precedes the severe headache (Borsook et al., 2015; Ravishankar \& Demakis, 2007; "The International classification of Headache disorders," 2013).
While migraine is clearly debilitating in the ictal period, in the last few years, many studies have focused on the evaluation of cognitive aspects observed in migraineurs during the interictal period, leading to various perspectives on the long-term effects of the disorder. Some investigations showed decreased cognitive skills while others did not. This includes research on specific processes such as executive functions $(\mathrm{EF})$, visual information processing, global impairment, attention, processing speed, and memory (Camarda, Monastero, Pipia, Recca, \& Camarda, 2007; Koppen et al., 2011; Le Pira et al., 2000; Mulder, Linssen, Passchier, Orlebeke, \& de Geus, 1999; Rist et al., 2012; Rist \& Kurth, 2013; Suhr \& Seng, 2012; Waldie, Hausmann, Milne, \& Poulton, 2002; Öze et al., 2016). For instance, EF such as planning, decision-making, problem-solving and behavioral inhibition in patients with chronic migraine showed decreased performance for migraine patients in all of these $\mathrm{EF}$, although statistically it was not significant for the Gambling Task (Rist et al., 2012).

Other research on patients suffering from white matter lesions and either MWA or MWoA evidenced lower performance on verbal fluency and EF for each migraine group (Le Pira et al., 2014). However, these researchers did not find a strong correlation with the presence of white matter lesions. Another study searched for the neurobiological bases of difficulties with EF in migraine patients, showing that they had lower grey matter density in the parietal and frontal lobes, which could explain the slow response and delayed processing speed in several different tasks (Schmitz et al., 2008). Similar to this was research that used neuroimaging and electrophysiological technologies to seek neurobiological explanations for the cognitive deficits and brain structure anomalies in migraine patients (Coppola et al., 2015; Liu et al., 2015; Mathur et al., 2015).

Highlighting the potential seriousness of migraine, it is possibly associated with neurological disorders, such as ischemia and dementia (Paemeleire, 2009; Santamarta et al., 2014; Sas, Párdutz, Toldi, \& Vécsei, 2010). Sas et al. (2010) considered that ischemia, dementia

| Universitas Psychologica | V. I5 | No.5 | 2016 | 
and migraine share common pathophysiological mechanisms related to mitochondrial function, and emphasized the higher prevalence of migraine, particularly MWA, in people who later suffered a stroke (Sas et al., 2010). In contrast, while Paemeleire (2009) noted that migraine was associated with certain brain abnormalities (especially cerebral white matter hyperintensity), he argued that it was a substantial risk factor for ischemia only when found in women below the age of 45 who were taking oral contraceptives, and was associated with cognitive decline only in periods during or close to attacks, rather than in the long term (Paemeleire, 2009).

Contrary to these findings, other studies showed no significant statistical differences when assessing processes like verbal and arithmetic problem solving, spatial problem solving, processing speed, vocabulary and language in interictal migraine patients versus control group performance (Baars, van Boxtel, $\&$ Jolles, 2010; Pearson, Chronicle, Maylor, \& Bruce, 2006). As mentioned above, research concerning cognitive impairment in migraine is still controversial and marked by inconsistent evidence, especially in the areas of visuospatial and constructional abilities, visual memory and reasoning, motor skills, and cognitive flexibility (Gil-Gouveia, Oliveira, \& Martins, 2015b; Suhr $\&$ Seng, 2012). Specifically, various studies have tried to establish a relation between migraine and memory (Calandre, Bembibre, Arnedo, \& Becerra, 2002; Gil-Gouveia et al., 2015b; Le Pira et al., 2000; Moutran et al., 2011). This research has shown evidence of deficits in visuo-spatial, verbal and working memory in people with migraine. Similar results have suggested deficits in visuospatial memory tasks and verbal performance with attentional deficits, taking into account that the tasks provided were generally oriented to evaluate memory performance through strategies and organization (Le Pira et al., 2000; Moutran et al., 2011).

All these results are difficult to compare or generalize because the study designs were different, samples of subjects were small, age ranges were very large, the tasks or neuropsychological tests used were different, and almost all studies were cross-sectional, with very few longitudinal studies. Moreover, these investigations have had inconclusive results when exploring specific memory issues such as encoding, consolidation and recall of visual and verbal information, which might lead to better identification of cognitive differences in migraine patients. Therefore, the principal aim of the current project was to use a variety of different memory tasks in a cross-sectional design, in order to identify differences in specific memory processes and the role of EF in memory during the interictal period, in people with MWA, with MWoA, and in a control group.

\section{Methods and Materials}

\section{Patients}

Twenty-six participants for the MWA group and nineteen participants for the MWoA group were selected based on the inclusion and exclusion criteria presented in Table 1 . This represented all patients attending the Neurology service of the Clínica Universidad de La Sabana (Chía, Colombia) in the years 2013-2015, who met the inclusion criteria and wished to take part in the research. The control group was formed by a similar number of 25 healthy individuals who had similar demographic characteristics to the patients. A chi-squared test and oneway ANOVA, respectively, revealed that the small differences in gender and age composition between groups were not significant.

\section{TABLE 1}

Description of inclusion and exclusion criteria of study

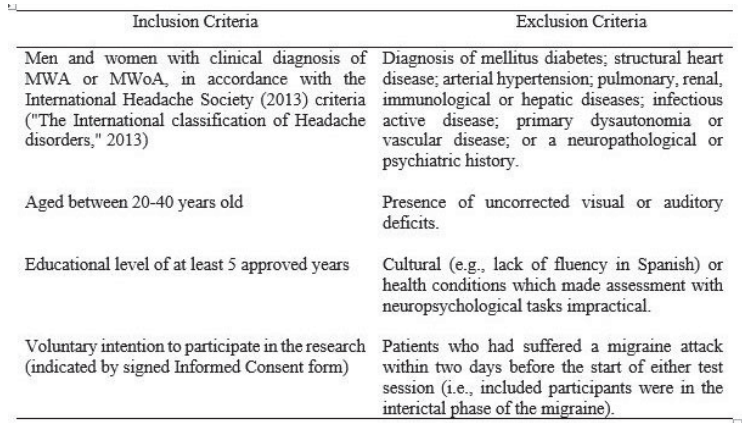




\section{Instruments}

For the assessment various neuropsychological tests were selected, as described in Table 2 . Exactly the same tests and study protocols were used for all three groups. An informed consent form was prepared and used to gain permission for the collection of sociodemographic data and medical history. The informed consent form and a description of the study protocol were presented to all participants before running the first assessment session.

\section{TABLE 2}

Description of Tests for Neuropsychological

Assessment

\begin{tabular}{|c|c|c|}
\hline Test & $\begin{array}{l}\text { Information obtained through } \\
\text { application test }\end{array}$ & Cognitive Processes assessed \\
\hline $\begin{array}{l}\text { Spain - Complutense Verbal } \\
\text { Learning Test (TAVEC) } \\
\text { (Garcia-Herranz, Diaz- } \\
\text { Mardomingo, \& Peraita, } \\
\text { 2014; Nieto et al., 2014) }\end{array}$ & $\begin{array}{l}\text { Immediate Memory (first trial) } \\
\text { Short-Term memory (first to fifth trial) } \\
\text { Long-Term Memory } \\
\text { Recognition } \\
\text { Proactive and Retroactive Interference } \\
\text { Semantic Association } \\
\text { Serial Association }\end{array}$ & $\begin{array}{l}\text { Working Memory } \\
\text { Encoding and consolidation } \\
\text { Recall } \\
\text { Recall } \\
\text { Executive functions (Inhibition) } \\
\text { Executive Strategies } \\
\text { Semantic declarative memory }\end{array}$ \\
\hline $\begin{array}{l}\text { Rey-Osterrieth Complex } \\
\text { Figure Test (ROCF) (Peña- } \\
\text { Casanova et al., 2009) }\end{array}$ & $\begin{array}{l}\text { Copy } \\
\text { Copy strategy } \\
\text { Evocation copy }\end{array}$ & $\begin{array}{l}\text { Visuocontructional skills } \\
\text { Executive functions (Planning) } \\
\text { Visual Memory (Recall) }\end{array}$ \\
\hline $\begin{array}{l}\text { Grober and Buschke Free } \\
\text { and Cued Selective } \\
\text { Reminding test (FCSRT) } \\
\text { (Clerici et al, 2015; Lemos, } \\
\text { Simoes, Santiago, \& } \\
\text { Santana, 2015; Zimmerman } \\
\text { et al., 2015) }\end{array}$ & $\begin{array}{l}\text { Reading } \\
\text { Encoding with semantic key } \\
\text { Immediate evocation } \\
\text { Free Recall } \\
\text { Facilitated Recall } \\
\text { Recognition Task }\end{array}$ & $\begin{array}{l}\text { Reading } \\
\text { Encoding } \\
\text { Memory } \\
\text { Working Memory } \\
\text { Encoding and consolidation } \\
\text { Evocated Recall } \\
\text { Facilitated evoked Recall }\end{array}$ \\
\hline
\end{tabular}

Source: own work.

\section{Study Protocol}

The study protocol had three phases. In the first phase, organization of the protocol and ethical approval were established by the Universidad de La Sabana. The second phase was the data collection. Participants were contacted from a database of patients who had attended the Neurology service of the Clinica Universidad de La Sabana (Chía, Colombia) and met the inclusion criteria but not the exclusion criteria for the study. Those who voluntarily agreed to participate in the research were scheduled for the assessment session. In this session (about 11/2 hours long) the informed consent was signed, and sociodemographic, clinical, and medical data were also collected. Then the application of the Spain - Complutense Verbal Learning Test (TAVEC), Rey-Osterrieth Complex Figure Test (ROCF), and Grober and Buschke Free and Cued
Selective Reminding test (FCSRT) tests took place (see Table 2 for details of these tests). Evaluation was performed using a blind strategy with two neuropsychologists who examined the participants without knowing their neurological diagnosis. After eight days, feedback was made to participants and they were given a final report with their general results. In the third phase, the database of results was digitized with the specific variables to be analyzed. In the database were the sociodemographic, clinical and medical data of each patient, and their scores for each neuropsychological task. With this information we began the statistical analysis, which is described in the following section.

\section{Measurements and statistical analysis}

All statistical analyses were carried out using SPSS for Windows, version 23.0. For all these analyses the one independent variable was considered to be clinical symptoms of migraine and had three levels: MWA, MWoA, and Control. The 18 dependent variables were encoding, consolidation, and recognition, serial and semantic association and retroactive and proactive interference measures in the TAVEC of verbal memory processing; recall and recognition measures in the FCSRT; direct and percentile copy strategy, direct and percentile recall, direct and percentile visuo-constructional skills, direct and percentile copy time, and direct recall time in the ROCF. Multivariate analysis of covariance (MANOVA) was first carried out on all dependent variables. Data were tested for normality with the Levene test, and variables that showed homogeneous variance across groups were analyzed using a series of univariate analyses of variance (ANOVAs). The Kruskal-Wallis nonparametric test was used for those variables that showed heterogeneous variance between groups. Where ANOVAs showed significant differences between groups, post hoc analyses were carried out using Tukey's test to identify the source of these differences. Next the two migraine groups were merged, and a series of Student's $t$ tests for independent samples (or the Mann-Whitney U 
test for variables showing heterogeneous variance between groups) were used to compare migraine patients as a whole with the control group.

\section{Results}

Demographic and clinical data for the three groups of participants are summarized in Table 3. Seven participants from the initial sample of seventy (three in the MWoA group, two in the MWA group, and two in the Control group) were excluded from the analysis either because they presented migraine attack within 48 hours of data collection (meaning that they were not unambiguously in the interictal period), or because they took the decision not to proceed with the study due to lack of time.

The remaining participants completed all tests, and thus there were no missing data in the analysis. The final sample was made up of 63 participants with a mean age of 25 years $(\mathrm{SD}=$ 5.77), distributed into MWA $(n=24)$, MWoA $(n=16)$, and Control $(n=23)$ groups.

\section{TABLE 3}

Demographic and clinical data for the three groups

\begin{tabular}{|c|c|c|c|c|c|c|}
\hline Demographic and clinical data & MWA & $\%$ & MWoA & $\%$ & Control & $\%$ \\
\hline \multicolumn{7}{|l|}{ Sex } \\
\hline male & 5 & 20.8 & 4 & 25 & 8 & 34.8 \\
\hline female & 19 & 79.2 & 12 & 75 & 15 & 65.2 \\
\hline \multicolumn{7}{|l|}{ Age } \\
\hline range & $20-40$ years & & $20-40$ years & & $20-40$ years & \\
\hline mean $=S D$ & $27 \pm 6.8$ & & $25 \pm 4.7$ & & $24 \pm 5$ & \\
\hline \multicolumn{7}{|l|}{ Marital status } \\
\hline single & 20 & 83.3 & 14 & 87.5 & 22 & 95.7 \\
\hline married & 3 & 12.5 & 2 & 12.5 & 1 & 4.3 \\
\hline free union & 1 & 4.2 & & & & \\
\hline \multicolumn{7}{|l|}{ Education years } \\
\hline 12 - 15 years (college student) & 13 & 54.2 & 8 & 50 & 18 & 78.3 \\
\hline $16-19$ years (professional) & 8 & 33.3 & 7 & 43.8 & 3 & 13 \\
\hline$>20$ years (postgraduate) & 3 & 12.5 & 1 & 6.3 & 2 & 8.7 \\
\hline \multicolumn{7}{|l|}{ Disease duration years } \\
\hline$\leq 5$ years & 1 & 95.8 & 8 & 50 & & \\
\hline$>5$ years & 23 & 4.2 & 8 & 50 & & \\
\hline \multicolumn{7}{|l|}{ Number attacks at year } \\
\hline \multirow{2}{*}{\multicolumn{6}{|c|}{ Attack duration hours }} & \\
\hline & & & & & & \\
\hline \multicolumn{7}{|l|}{$\begin{array}{l}\text { meant } \mathrm{SD} \\
\text { Pharmacotherapy }\end{array}$} \\
\hline acute & 21 & 88 & 12 & 75 & & \\
\hline acute-prophylactic & 2 & 8 & 2 & 12.5 & & \\
\hline neither & 1 & 4 & 2 & 12.5 & & \\
\hline \multicolumn{7}{|l|}{ Type of aura } \\
\hline visual & & & 13 & 81.3 & & \\
\hline tactile & & & 3 & 18.7 & & \\
\hline
\end{tabular}

Source: own work.

An initial MANOVA revealed a significant effect of migraine type on the dependent variables taken as a whole $\left(\mathrm{F}_{(2,62)}=1.624\right.$, $p=0.033$, Wilk's $\lambda=0.311$ ). A series of follow-up ANOVAs were then carried out on those variables for which Levene's test showed homogeneous variance between groups (all dependent variables except the TAVEC verbal memory recognition measure, ROCF direct copy and time spent measures, and ROCF percentile recall measure). Because a total of 18 dependent variables were being tested in this analysis, a corrected Bonferroni $\alpha$ value of 0.0025 was used to establish statistical significance instead of the conventional value of 0.05 .

ANOVAs conducted on the data from the verbal memory measures did not reveal significant differences between groups in the encoding, consolidation, association or interference measures of the TAVEC test, or the recall or recognition measures in the FCSRT (all $F<2.5$, all $p>0.05$ ). Finally, in the ROCF, the one result that reached significance with the corrected $\alpha$ value was percentile copy strategy $\left(\mathrm{F}_{(2,62)}=14.7, p<0.001, \eta^{2}=0.328 ;\right.$ Figure 1$)$. The results that approached significance for the ROCF test were measures of direct recall $\left(\mathrm{F}_{(2,62)}\right.$ $\left.=6.44, p=0.003, \eta^{2}=0.177\right)$ and of direct and percentile visuo-constructional skills $\left(\mathrm{F}_{(2,62)}\right.$ $=4.27, p=0.018, \eta^{2}=0.125 ; \quad F_{2,62)}=4.65, p$ $\left.=0.013, \eta^{2}=0.134\right)$. There were no significant differences for the ROCF direct and percentile copy time measures $\left(\mathrm{F}_{(2,62)}<1, p>0.05\right)$. 


\section{Figure 1}

The data of three groups in the copy strategy of ROCF

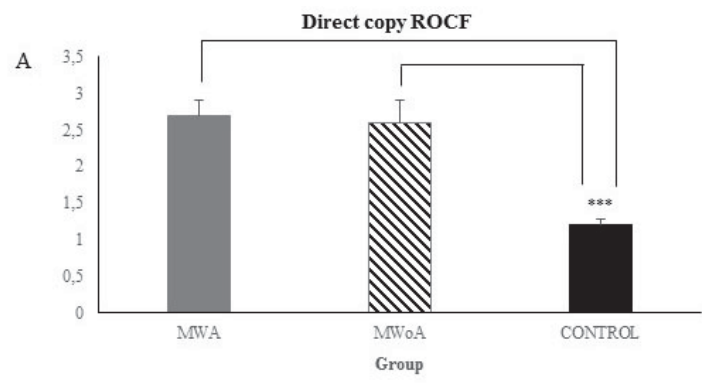

B

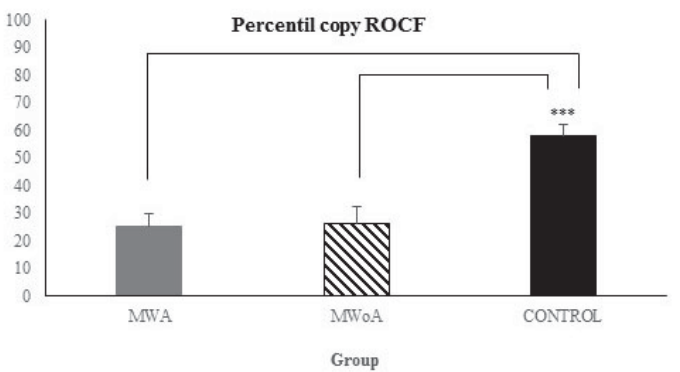

There were significant differences between groups in direct $(p<0.001)(\mathrm{A})$ and percentile copy strategy $(p<0.001)(B)$. Tukey post hoc tests indicated that differences were between control and both migraine groups, control with MWA ( $p$ $<0.001)$, and control with MWoA $(p<0.001)$.

Source: own work.

The dependent variables that showed heterogeneous variance between groups were analyzed with the Kruskal-Wallis non-parametric test. This found no significant differences between groups in the TAVEC verbal recognition memory test or the time recall measure of the ROCF test. However, there was a significant difference for ROCF direct copying strategy $\left(\chi^{2}\right.$ $=21.7, p<0.001, \eta^{2}=0.35$; see Figure $1)$, and a result that approached significance for ROCF percentile recall $\left(\chi^{2}=10.8, p=\right.$ 0.004, $\left.\eta^{2}=0.174\right)$. Figure 2 illustrates some qualitative differences in ROCF performance between groups.

\section{Figure 2}

Significant sample execution ROCF of each group

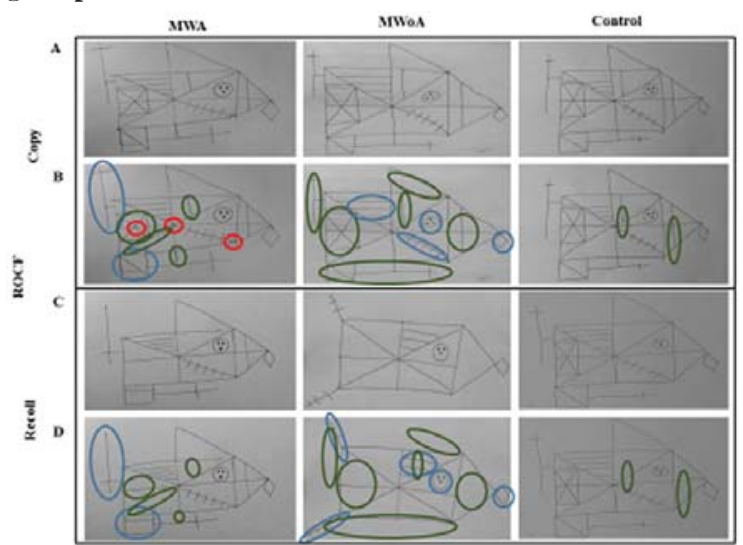

ROCF copy (A), evaluation ROCF copy (B), ROCF recall (C), and evaluation ROCF recall (D). As can be seen, it shows an increase in amount of mistakes done for migraine groups. MWoA shows a worse performance and made more visoespatial mistakes including rotation and more omissions than MWA. Control group made fewer mistakes than both migraine groups. Blue color: visoespatial mistakes, green color: omissions, red color: planning mistakes. Source: own work.

In order to provide more statistical power, and because post hoc analysis showed no differences between migraine groups but significant differences between each of them and the control group, the two migraine groups were merged, and then compared with the control group using Student's $t$ test for independent samples (for variables showing homogeneous variance) and the Mann-Whitney $U$ test (for those showing heterogeneous variance). Again using a corrected $\alpha$ level of 0.0025 , results (Table 4) showed significant, large differences in ROCF percentile copy strategy $\left(\mathrm{t}_{(61)}=5.46, p<0.001\right.$, $d=1.46)$, and direct recall $\left(\mathrm{t}_{(61)}=3.28, p=\right.$ $0.002, d=0.952)$. The Mann-Whitney $U$ test found significant differences in ROCF direct copy strategy $(U=154, p<0.001, r=0.586)$ and percentile recall $(U=231, p=0.001, r=0.414)$. 


\section{TABLE 4}

Descriptive data of Neuropsychological variables in merged group with migraine compared to control group

\begin{tabular}{|c|c|c|c|c|}
\hline Neuropsychological Test & $\begin{array}{c}\text { Mean } \neq \text { SD } \\
\text { Migraine }\end{array}$ & $\begin{array}{c}\text { Mean } \neq \text { SD } \\
\text { Control }\end{array}$ & $T$ & $P$ \\
\hline TAVEC & & & & \\
\hline Encoding & $23.5 \pm 11.1$ & $22.1 \pm 11.5$ & 0.489 & 0.627 \\
\hline Consolidation & $58.7 \pm 7.8$ & $57.4 \pm 9.3$ & 0.555 & 0.581 \\
\hline Recognition & $15.4 \pm .7$ & $15.2 \pm 1.1$ & 0.984 & 0.329 \\
\hline Serial association & $5.63 \pm 40.0$ & $3.91 \pm 2.9$ & 1.686 & 0.193 \\
\hline Semantic association & $16.9 \pm 6.9$ & $16.4 \pm 6.7$ & 0.363 & 0.363 \\
\hline Retroactive interference & $-.25 \pm 1.8$ & $-.13 \pm 2.2$ & -0.228 & 0.820 \\
\hline $\begin{array}{l}\text { Proactive interference } \\
\text { CCRT }\end{array}$ & $-.28 \pm 1,1$ & $-.48 \pm 1.7$ & 0.564 & 0.575 \\
\hline FCSRT & & & & \\
\hline Recall & $10.7 \pm 5.5$ & $10.2 \pm 3.2$ & 0.380 & 0.706 \\
\hline $\begin{array}{l}\text { Recognition } \\
\text { ROCF }\end{array}$ & $15.9 \pm .1$ & $15.9 \pm .2$ & 0.397 & 0.693 \\
\hline $\begin{array}{l}\text { ROCF } \\
\text { Direct Copy strategy ... }\end{array}$ & & & & \\
\hline $\begin{array}{l}\text { Direct Copy strategy } \\
\text { Percentile Copy strategy }\end{array}$ & $\begin{array}{l}2.7 \pm 1.2 \\
25.239\end{array}$ & $\begin{array}{l}1.2 \pm 4 \\
57.5 \pm 202\end{array}$ & 5.518 & 0.000 \\
\hline $\begin{array}{l}\text { Percentile Copy strategy } \\
\text { Direct Recall }\end{array}$ & $\begin{array}{l}25.2 \pm 23.9 \\
22.6 \pm 5\end{array}$ & $\begin{array}{l}77.5 \pm 20.2 \\
27 \pm 4.2\end{array}$ & $\begin{array}{l}-5.456 \\
-3.531\end{array}$ & 0.000 \\
\hline Percentile Recall $\cdots$ & $59.5 \pm 28.3$ & $81.1 \pm 18.3$ & $\begin{array}{l}-3.531 \\
-3.277\end{array}$ & 0.002 \\
\hline Direct Visuoconstructional Skills & $30.8 \pm 2.6$ & $32.8 \pm 3$ & -2.708 & 0.009 \\
\hline Percentile Visuoconstructional Skills & $57.4 \pm 22$ & $73.8 \pm 23.9$ & -2.760 & 0.008 \\
\hline Direct Time Copy & $182.7 \pm 129.1$ & $178.9 \pm 60.6$ & 0.131 & 0.896 \\
\hline Percentile Time Copy & $\begin{array}{l}55.2 \pm 24.1 \\
1305+38\end{array}$ & $52.1 \pm 21.2$ & 0.513 & 0.610 \\
\hline
\end{tabular}

\section{Discussion}

The aim of this research was to identify differences in memory processes and the role of EF in these processes, between interictal patients with MWA, those with MWoA, and healthy controls.

Differences were observed in execution of the ROCF in copy strategy and the visual memory task but not in other main scores such as visualconstructional skills or execution time. These findings could be interpreted in terms of migraine not having a direct influence on constructional abilities or processing speed (Moutran et al., 2011).

One possible explanation for the strong effects on ROCF task performance in copy strategy and recall scores is that the ROCF is a relatively complex task sometimes associated with to EF

(Nakano et al., 2006; Ogino et al., 2009; Watanabe et al., 2005). Thus, the task has been argued to reflect EF such as organizational and planning abilities, and not only visual cognitive functions (Ogino et al., 2009; Watanabe et al., 2005). ROCF might therefore be differentiated from other memory measures due to its sensitivity to different cognitive domains, notably EF. Because of the complexity of the figure involved, organizational strategies are needed to recognize the presence and configuration of the figure elements, and then to draw or recall them. It is precisely these aspects which were found altered in this research. These findings are therefore suggestive of the involvement of EF in migraineurs' cognitive profile (Schmitz et al., 2008).

According to these results, migraineurs could be impaired in visual memory performance because of a weakening in some complex EF applied to the visuospatial encoding process, as measured by their copy strategy and recall scores. This alteration may make it difficult to consolidate visual information, without apparently influencing memory for verbal information. This is consistent with previous research that exposed EF alterations in migraine patients (Le Pira et al., 2000). However, it is important to note that no differences were found between the two migraine groups or between sexes. Apparently, any differences in cognitive processes in people with or without aura, and especially in females and males, are more related to pain intensity during migraine attacks and to the role of sex hormones (Faria et al., 2015; Finocchi \& Strada, 2014; Gil-Gouveia, Oliveira, \& Martins, 2015a; Le Pira et al., 2000; Maleki et al., 2012; Suhr \& Seng, 2012; Wang et al., 2014).

Another possible explanation for the results stems from differences between measures that involve visual or verbal information, especially if these tasks require processes such as working memory (Camarda et al., 2007; Öze et al., 2016). The data obtained in visual processing may exhibit lower performance than verbal processing in migraine patients. These findings could be explained by the stability of verbal memory performance across time because verbal skills are supported by schooling and acculturation (Horn \& Cattell, 1966). Verbal skills have been described as the most resistant cognitive skills when assessed after certain brain lesions associated with migraine (Paemeleire, 2009).

This difference could be related to the distinction between crystallized and fluid intelligence, since verbal tasks have mostly been associated with crystallized intelligence (Gil-Gouveia et al., 2015a; Horn \& Cattell, 1966). Therefore, verbal abilities could be 
more resistant to the impact of migraine in subjects who are suffering it. This would explain the absence of a difference between migraine and non-migraine groups on the TAVEC and FCSRT tasks. In contrast, since performance on visual tasks is associated with fluid intelligence, this might change more easily when cerebral alterations occur (Duncan, Burgess, \& Emslie, 1995; Friedman et al., 2006; van Aken, Kessels, Wingbermühle, van der Veld, \& Egger, 2015). This could thus be an explanation for the observed findings about copy strategy and recall in the ROCF

Although the participation of EF in cognitive profiles has been widely described, other variables might intervene in these results. For example, level of education and occupational profile can affect the results obtained in the neuropsychological assessment of participants. However, the current study does not evidence any impact of these variables on the cognitive profiles of either migraine patients or control participants, in line with other studies in this area (e.g., Gil-Gouveia, Oliveira, \& Martins, 2015b). Regarding the evaluation of measures of verbal memory encoding, consolidation and recall the results obtained in this investigation did not demonstrate significant differences between groups. These findings are also consistent with existing studies of migraineurs (Gaist et al., 2005; Kececi \& Atakay, 2009). However, they contradict other literature that suggested possible differences in certain cognitive processes, including verbal memory, only in people with MWoA (Le Pira et al., 2000).

Indeed, one limitation of this study relates to the diagnostic criteria of MWoA. These criteria remain unclear, because this type of migraine can be confused with other kinds of headaches, such as tension headache. This could create bias if large numbers of participants without genuine migraine were included in the study. However, in this respect it is worth noting that no significant differences were found between participants with MWA and those with MWoA. Additional limitations arise from the assessment protocol. It was designed to assess memory initially, but we interpreted the significant results on some memory tasks, and insignificant results on others, in terms of a differential involvement of EF. In future, EF-specific tasks could be integrated as part of the assessment protocol, to clarify the interrelationships between memory, EF, and migraine. Furthermore, larger samples should be used, in order to increase statistical power and analyze age and other demographic effects.

In conclusion, the main results of this study suggest that people with migraine (with or without aura) have attenuated EF necessary for complex visual memory in the ROCF. The differences between control and migraine groups in both copy strategy and recall performance in the ROCF indicate the existence of brain dysfunction in people with MWA and MWoA, which affects the use of EF — such as encoding, planning and organization - that are recruited in visual-constructional memory tasks. Our results may help to resolve inconsistencies in the literature, by possibly explaining why some studies have found a relationship between migraine and memory performance, but others have not. In this regard it would be worth systematically reviewing previous memory studies to analyze whether the type of task used had any effect on the detection of this relationship. Such a project could clarify the particular cognitive deficits associated with migraine, and ultimately inform interventions that improve migraine sufferers' functional outcomes and quality of life.

\section{Acknowledgement}

This research was supported by funds provided by the Universidad de La Sabana, Bogotá, Colombia.

\section{References}

Baars, M. A., van Boxtel, M. P., \& Jolles, J. (2010). Migraine does not affect cognitive decline: results from the Maastricht aging study. Headache, 50(2), 176-184. doi:10.1111/j.1526-4610.20090.01572.x

Borsook, D., Maleki, N., \& Burstein, R. (2015). Migraine. In M. J. Zigmond, J. T. Coyle, 
\& L. Rowland (Eds.), Neurobiology of Brain Disorders (pp. 693-708). San Diego: Academic Press.

Bussone, G. (2004). Pathophysiology of migraine. Neurol Sci, 25 Suppl 3, S239-241. doi:10.1007/s10072-004-0295-3

Calandre, E. P., Bembibre, J., Arnedo, M. L., \& Becerra, D. (2002). Cognitive disturbances and regional cerebral blood flow abnormalities in migraine patients: their relationship with the clinical manifestations of the illness. Cephalalgia, 22(4), 291-302.

Camarda, C., Monastero, R., Pipia, C., Recca, D., \& Camarda, R. (2007). Interictal executive dysfunction in migraineurs without aura: relationship with duration and intensity of attacks. Cephalalgia, 27(10), 1094-110. doi:10.1111/j.1468-2982.20070.01394.x

Clerici, F., Ghiretti, R., Di Pucchio, A., Pomati, S., Cucumo, V., Marcone, A., Cappa, S. F. (2015). Construct validity of the Free and Cued Selective Reminding Test in older adults with memory complaints. J Neuropsychol. doi:10.1111/jnp.12087

Coppola, G., Di Renzo, A., Tinelli, E., Iacovelli, E., Lepre, C., Di Lorenzo, C., Pierelli, F. (2015). Evidence for brain morphometric changes during the migraine cycle: a magnetic resonance-based morphometry study. Cephalalgia, 35(9), 783-791. doi:10.1177/0333102414559732

Duncan, J., Burgess, P., \& Emslie, H. (1995). Fluid intelligence after frontal lobe lesions. Neuropsychologia, 33(3), 261-268.

Faria, V., Erpelding, N., Lebel, A., Johnson, A., Wolff, R., Fair, D., Borsook, D. (2015). The migraine brain in transition: girls vs boys. Pain, 156(11), 2212-2221. doi:10.1097/ j.pain 0.0000000000000292

Finocchi, C., \& Strada, L. (2014). Sex-related differences in migraine. Neurol Sci, 35 Suppl 1, 207-213. doi:10.1007/s10072-014-1772$\mathrm{y}$

Friedman, N. P., Miyake, A., Corley, R. P., Young, S. E., Defries, J. C., \& Hewitt, J. K. (2006). Not all executive functions are related to intelligence. Psychol Sci, 17(2), 172-179. doi:10.1111/j.1467-9280.20060.01681.x

Gaist, D., Pedersen, L., Madsen, C., Tsiropoulos, I., Bak, S., Sindrup, S., Christensen, K. (2005). Longterm effects of migraine on cognitive function: a population-based study of Danish twins. Neurology, 64(4), 600-607. doi:10.1212/01.WNL0.0000151858.15482.66

Garcia-Herranz, S., Diaz-Mardomingo, M. C., \& Peraita, H. (2014). Evaluation and follow-up of healthy aging and aging with mild cognitive impairment (MCI) through TAVEC. Anales De Psicologia, 30(1), 373-380. doi:10.6018/ analesps.30.1.150711

Gil-Gouveia, R., Oliveira, A. G., \& Martins, I. P. (2015a). Assessment of cognitive dysfunction during migraine attacks: a systematic review. J Neurol, 262(3), 654-665. doi: 10.1007/s00415-014-7603-5

Gil-Gouveia, R., Oliveira, A. G., \& Martins, I. P. (2015b). Sequential brief neuropsychological evaluation of migraineurs is identical to controls. Acta Neurol Scand. doi:10.1111/ane.12530

Harmon, T. P. (2015). Diagnosis and management of migraines and migraine variants. Prim Care, 42(2), 233-241. doi:10.1016/j.pop.20150.010.003

Horn, J. L., \& Cattell, R. B. (1966). Refinement and test of the theory of fluid and crystallized general intelligences. J Educ Psychol, 57(5), 253-270.

Kececi, H., \& Atakay, S. (2009). Effects of topiramate on neurophysiological and neuropsychological tests in migraine patients. Journal of Clinical Neuroscience, 16(12), 1588-1591.

Koppen, H., Palm-Meinders, I., Kruit, M., Lim, V., Nugroho, A., Westhof, I., Hommel, B. (2011). The impact of a migraine attack and its after-effects on perceptual organization, attention, and working memory. Cephalalgia, 31(14), 1419-1427. doi: $10.1177 / 0333102411417900$

Le Pira, F., Reggio, E., Quattrocchi, G., Sanfilippo, C., Maci, T., Cavallaro, T., \& 
Zappia, M. (2014). Executive dysfunctions in migraine with and without aura: what is the role of white matter lesions? Headache, 54(1), 125-130. doi:10.1111/head.12158

Le Pira, F., Zappalà, G., Giuffrida, S., Lo Bartolo, M. L., Reggio, E., Morana, R., \& Lanaia, F. (2000). Memory disturbances in migraine with and without aura: a strategy problem? Cephalalgia, 20(5), 475-478.

Lemos, R., Simoes, M. R., Santiago, B., \& Santana, I. (2015). The free and cued selective reminding test: Validation for mild cognitive impairment and Alzheimer's disease. Journal of Neuropsychology, 9(2), 242-257. doi:10.1111/jnp.12048

Liu, J., Zhao, L., Lei, F., Zhang, Y., Yuan, K., Gong, Q., Tian, J. (2015). Disrupted resting-state functional connectivity and its changing trend in migraine suffers. Hum Brain Mapp, 36(5), 1892-1907. doi:10.1002/hbm.22744

Maleki, N., Linnman, C., Brawn, J., Burstein, R., Becerra, L., \& Borsook, D. (2012). Her versus his migraine: multiple sex differences in brain function and structure. Brain, 135(Pt 8), 2546-2559. doi:10.1093/brain/ aws 175

Mathur, V. A., Khan, S. A., Keaser, M. L., Hubbard, C. S., Goyal, M., \& Seminowicz, D. A. (2015). Altered cognition-related brain activity and interactions with acute pain in migraine. Neuroimage Clin, 7, 347-358. doi:10.1016/j.nicl.20150.010.003

Moutran, A. R., Villa, T. R., Diaz, L. A., Noffs, M. H., Pinto, M. M., Gabbai, A. A., \& Carvalho, D. e. S. (2011). Migraine and cognition in children: a controlled study. Arq Neuropsiquiatr, 69(2A), 192-195.

Mulder, E. J., Linssen, W. H., Passchier, J., Orlebeke, J. F., \& de Geus, E. J. (1999). Interictal and postictal cognitive changes in migraine. Cephalalgia, 19(6), 557-565; discussion 541.

Nakano, K., Ogino, T., Watanabe, K., Hattori, J., Ito, M., Oka, M., \& Ohtsuka, Y. (2006). A developmental study of scores of the Boston Qualitative Scoring System. Brain Dev, 28(10), 641-648. doi:10.1016/ j.braindev.20060.040.011
Nieto, A., Hernandez-Rodriguez, E., HernandezTorres, A., Velasco Rodriguez-Solis, P., Hess-Medler, S., Machado-Fernandez, A., . . . Barroso, J. (2014). Alternate form of the test de aprendizaje verbal Espana-Complutense (TAVEC). Revista De Neurologia, 58(9), 389-395.

Ogino, T., Watanabe, K., Nakano, K., Kado, Y., Morooka, T., Takeuchi, A., Ohtuska, Y. (2009). Predicting executive function task scores with the Rey-Osterrieth Complex Figure. Brain and Development, 31 (1), 52 57.

Paemeleire, K. (2009). Brain lesions and cerebral functional impairment in migraine patients. J Neurol Sci, 283(1-2), 134-136. doi: 10.1016/j.jns.20090.02.333

Pearson, A. J., Chronicle, E. P., Maylor, E. A., \& Bruce, L. A. (2006). Cognitive function is not impaired in people with a long history of migraine: a blinded study. Cephalalgia, 26(1), 74-80. doi:10.1111/ j.1468-2982.20050.01001.x

Peña-Casanova, J., Gramunt-Fombuena, N., Quinones-Ubeda, S., Sanchez-Benavides, G., Aguilar, M., Badenes, D., Team, N. S. (2009). Spanish Multicenter Normative Studies (NEURONORMA Project): Norms for the Rey-Osterrieth Complex Figure (Copy and Memory), and Free and Cued Selective Reminding Test. Archives of Clinical Neuropsychology, 24(4), 371-393. doi:10.1093/arclin/acp041

Ravishankar, N., \& Demakis, G. J. (2007). The neuropsychology of migraine. Dis Mon, 53(3), 156-161. doi:10.1016/ j.disamonth.20070.040.006

Rist, P. M., Kang, J. H., Buring, J. E., Glymour, M. M., Grodstein, F., \& Kurth, T. (2012). Migraine and cognitive decline among women: prospective cohort study. BMJ, 345, e 5027.

Rist, P. M., \& Kurth, T. (2013). Migraine and cognitive decline: a topical review. Headache, 53(4), 589-598. doi:10.1111/ head.12046

Santamarta, E., Meilán, A., Saiz, A., Larrosa, D., Cernuda-Morollón, E., Calleja, S., 
Pascual, J. (2014). Chronic migraine does not increase posterior circulation territory (PCT) infarct-like lesions. J Neurol Sci, 336(1-2), 180-183. doi:10.1016/ j.jns.2013.10.035

Sas, K., Párdutz, A., Toldi, J., \& Vécsei, L. (2010). Dementia, stroke and migraine-some common pathological mechanisms. J Neurol Sci, 299(1-2), 55-65. doi:10.1016/ j.jns.2010.080.001

Schmitz, N., Arkink, E. B., Mulder, M., Rubia, K., Admiraal-Behloul, F., Schoonman, G. G., van Buchem, M. A. (2008). Frontal lobe structure and executive function in migraine patients. Neurosci Lett, 440(2), 92-96. doi:10.1016/j.neulet.20080.050.033

Suhr, J. A., \& Seng, E. K. (2012). Neuropsychological functioning in migraine: clinical and research implications. Cephalalgia, 32(1), 39-54. doi: $10.1177 / 0333102411430265$

The International classification of Headache disorders. (2013). (3rd edition ed., Vol. 33, pp. 629-808): Cephalalgia.

van Aken, L., Kessels, R. P., Wingbermühle, E., van der Veld, W. M., \& Egger, J. I. (2015). Fluid intelligence and executive functioning more alike than different? Acta Neuropsychiatr, 1-7. doi:10.1017/ neu.2015.46

Waldie, K. E., Hausmann, M., Milne, B. J., \& Poulton, R. (2002). Migraine and cognitive function: a life-course study. Neurology, 59(6), 904-908.

Wang, R., Dong, Z., Chen, X., Zhang, M., Yang, F., Zhang, X., Yu, S. (2014). Gender differences of cognitive function in migraine patients: evidence from event-related potentials using the oddball paradigm. J Headache Pain, 15, 6. doi:10.1186/1129-2377-15-6

Watanabe, K., Ogino, T., Nakano, K., Hattori, J., Kado, Y., Sanada, S., \& Ohtsuka, Y. (2005). The Rey-Osterrieth Complex Figure as a measure of executive function in childhood. Brain and Development, 27 (8), $564-569$.
Zimmerman, M. E., Katz, M. J., Wang, C., Burns, L. C., Berman, R. M., Derby, C. A., Lipton, R. B. (2015). Comparison of "Word" vs. "Picture" version of the Free and Cued Selective Reminding Test (FCSRT) in older adults. Alzheimer's \&ु Dementia: Diagnosis, Assessment $\mathbb{E}$ Disease Monitoring, 1(1), 94-10. doi: http://dx.doi.org/10.1016/ j.dadm.2014.110.006

Öze, A., Nagy, A., Benedek, G., Bodosi, B., Kéri, S., Pálinkás, É., . . . Braunitzer, G. (2016). Acquired equivalence and related memory processes in migraine without aura. Cephalalgia. doi: $10.1177 / 0333102416651286$

\section{Notes}

* Research article. 